\title{
Análise epidemiológica dos casos novos de tuberculose em uma população privada de liberdade no Nordeste brasileiro
}

Epidemiological analysis of new tuberculosis cases in a private freedom population in northeast Brazil

Análisis epidemiológico de nuevos casos de tuberculosis en una población privada de libertad en el Noreste de Brasil

Robert Lincoln Barros Melo

ORCID: https://orcid.org/0000-0002-3430-4411 Universidade Federal de Alagoas, Brasil

E-mail: robert.lincoln.b@gmail.com

Amuzza Aylla Pereira dos Santos

ORCID: https://orcid.org/0000-0001-6299-7190

Universidade Federal de Alagoas, Brasil

E-mail: amuzzasantos@bol.com.br

Clodis Maria Tavares

ORCID: https://orcid.org/0000-0001-6804-3064

Universidade Federal de Alagoas, Brasil

E-mail: clodistavares@yahoo.com.br

Erlon Oliveira dos Santos

ORCID: https://orcid.org/0000-0001-8489-5240

Secretaria Municipal de Saúde de Penedo, Brasil

E-mail: erlon.medtropical@hotmail.com

Valéria Bezerra Santos

ORCID: https://orcid.org/0000-0002-3026-9823

Secretaria de Estado da Saúde de Alagoas, Brasil

E-mail: valeria.santos67@gmail.com

Wanderlei Barbosa dos Santos

ORCID: https://orcid.org/0000-0001-9813-8857

Universidade Federal de Alagoas, Brasil

E-mail: wanderlei.santos@esenfar.ufal.br

\section{Resumo}

Objetivo: Analisar as características epidemiológicas dos casos novos de tuberculose entre as pessoas privadas de liberdade notificadas em Alagoas no período de 2009 a 2018. Método: Trata-se de um estudo epidemiológico descritivo, retrospectivo de abordagem quantitativa. A população do estudo foi composta por pessoas privadas de liberdade do estado de Alagoas notificadas com tuberculose no Sistema de Informação de Agravos de Notificação (SINAN) no período de 2009 a 2018. Os dados explicitados foram analisados por meio da estatística descritiva, com base na frequência relativa e absoluta em percentual. Resultados: Observou-se a elevação do número de casos novos de tuberculose no sistema prisional são eles 22,9\% (2013), 9,3\% (2016) e 13,4\% (2018). Dentre os 411 casos novos, constata-se que $80,8 \%$ eram do sexo masculino, na faixa etária de 20 e 39 anos $(63,5 \%)$. Em relação à raça/cor da pele, $330(80,3 \%)$ eram da cor preta/pardos e 11,4\% eram brancos. Em relação às características clínicas dos casos novos de tuberculose, a forma clínica pulmonar esteve quase que na totalidade, representada em $90,3 \%$ dos casos. Conclusão: Os resultados deste estudo levam à reflexão sobre a necessidade de melhoria na qualidade do cuidado prestado e a considerar que se continuarmos com o crescimento acelerado da população privada de liberdade, se não lançarmos estratégias específicas no contexto prisional e tratarmos os fatores de risco para adoecimento da tuberculose, será difícil pensar no fim da tuberculose.

Palavras-chave: Tuberculose; Prisioneiros; Populações vulneráveis; Saúde pública; Estudos epidemiológicos.

\section{Abstract}

Objective: To analyze the epidemiological characteristics of new cases of tuberculosis among people deprived of liberty reported in Alagoas from 2009 to 2018. Method: This is a descriptive, retrospective epidemiological study with a quantitative approach. The study population consisted of people deprived of liberty in the state of Alagoas notified with tuberculosis in the Notifiable Diseases Information System (SINAN) from 2009 to 2018. The data explained 
were analyzed using descriptive statistics, based on relative and absolute frequency in percentage. Results: There was an increase in the number of new cases of tuberculosis in the prison system: $22.9 \%$ (2013), 9.3\% (2016) and 13.4\% (2018). Among the 411 new cases, $80.8 \%$ were male, aged between 20 and 39 years $(63.5 \%)$. Regarding race/skin color, $330(80.3 \%)$ were black/brown and $11.4 \%$ were white. Regarding the clinical characteristics of new cases of tuberculosis, the pulmonary clinical form was almost entirely, represented in $90.3 \%$ of cases. Conclusion: The results of this study lead to reflection on the need to improve the quality of care provided and to consider that if we continue with the accelerated growth of the population deprived of liberty, if we do not launch specific strategies in the prison context and treat risk factors for tuberculosis illness, it will be difficult to think about the end of tuberculosis.

Keywords: Tuberculosis; Prisoners; Vulnerable populations; Public health; Epidemiologic studies.

\section{Resumen}

Objetivo: Analizar las características epidemiológicas de los nuevos casos de tuberculosis en personas privadas de libertad notificados en Alagoas de 2009 a 2018. Método: Se trata de un estudio epidemiológico descriptivo, retrospectivo, con abordaje cuantitativo. La población de estudio estuvo conformada por personas privadas de libertad en el estado de Alagoas notificadas con tuberculosis en el Sistema de Información de Enfermedades Notificables (SINAN) de 2009 a 2018. Los datos explicados fueron analizados mediante estadística descriptiva, con base en frecuencia relativa y absoluta en porcentaje. Resultados: Hubo un aumento en el número de nuevos casos de tuberculosis en el sistema penitenciario: 22,9\% (2013), 9,3\% (2016) y 13,4\% (2018). De los 411 casos nuevos, el $80,8 \%$ eran varones, con edades comprendidas entre los 20 y los 39 años (63,5\%). En cuanto a raza / color de piel, $330(80,3 \%)$ eran negros / marrones y el 11,4\% eran blancos. En cuanto a las características clínicas de los nuevos casos de tuberculosis, la forma clínica pulmonar estuvo casi en su totalidad, representada en el 90,3\% de los casos. Conclusión: Los resultados de este estudio llevan a reflexionar sobre la necesidad de mejorar la calidad de la atención brindada y a considerar que, si continuamos con el crecimiento acelerado de la población privada de libertad, si no ponemos en marcha estrategias específicas en el contexto penitenciario y tratar los factores de riesgo de la enfermedad de tuberculosis, será difícil pensar en el fin de la tuberculosis.

Palabras clave: Tuberculosis; Prisioneros; Poblaciones vulnerables; Salud pública; Estudios epidemiológicos.

\section{Introdução}

A tuberculose (TB) caracteriza-se por ser uma doença infecciosa cujo agente infeccioso é o Mycobacterium Tuberculosis, conhecido também como bacilo de Koch. A sua transmissão se dá através do ar, pela emissão perdigotas, de pessoa a pessoa, sendo que a tosse se caracteriza como o principal meio propagador (Araújo, et al., 2005).

A TB persiste como importante e desafiador problema no âmbito da saúde da população, contribuindo para manutenção do quadro de desigualdade e exclusão social em diversos países. É uma das enfermidades mais prevalentes entre as pessoas em situação de pobreza no mundo com elevada carga em termos de mortalidade, juntamente com o HIV/aids e a malária (Brasil, 2014).

Em geral, o quadro clínico desses pacientes se apresenta com um comprometimento do estado geral, febre baixa, geralmente vespertina, além de episódios de sudorese noturna, inapetência e emagrecimento, dor torácica e tosse, inicialmente seca, e quando produtiva acompanhada ou não de escarros hemoptoicos (Brasil, 2019).

A distribuição do número de casos ocorre de forma desigual no mundo, concentrando-se nos grupos sociais desfavorecidos tais com: pessoas em situação de pobreza e fome; pessoas privadas de liberdade; minorias étnicas (como os indígenas no Brasil) e aquelas vivendo com HIV/Aids. A tuberculose, além de decorrente, é também perpetuadora da pobreza, pois compromete a saúde dos indivíduos e suas famílias causando impactos econômicos e sociais (Brasil, 2014; Hargreaves, et al., 2011; WHO, 2014).

As pessoas privadas de liberdade são, em sua maioria, oriundas dos segmentos da população mais afetados pela tuberculose: homens jovens, com baixa escolaridade, desempregados ou empregados com baixa remuneração, que vivem em comunidades desfavorecidas das grandes cidades e algumas vezes nas ruas. Com frequência, têm antecedentes de tuberculose e de encarceramento, além de história de uso de drogas e taxa de infecção pelo HIV mais elevada do que a da população geral (Brasil, 2019). 
No país, a taxa de incidência da tuberculose na população prisional é cerca de 28 vezes superior à da população geral (Navarro et al., 2016). O sistema prisional em Alagoas não difere da realidade das demais regiões do país, pois a qualidade de vida e saúde é precária, por lotação de celas, contato próximo entre as pessoas que permitem a transmissão de agentes patológicos, a exemplo, a tuberculose (Silva et al., 2021). Desta forma, o estudo traz como objetivo analisar as características epidemiológicas dos casos novos de tuberculose entre as pessoas privadas de liberdade notificadas em Alagoas no período de 2009 a 2018.

\section{Metodologia}

Trata-se de um estudo de caráter epidemiológico descritivo, retrospectivo de abordagem quantitativa, realizada a partir dos dados secundários do Sistema de Informação de Agravos de Notificação (SINAN). Os dados foram coletados no primeiro semestre de 2020, a partir das informações disponíveis nos sites TABNET/DATASUS do Ministério da Saúde.

Delimitou-se a amostra ao período de 2009 a 2018. Os dados oficiais de 2019 ainda não foram disponibilizados pelo Ministério da Saúde.

As variáveis estudadas foram sexo, faixa etária, raça, escolaridade, beneficiário do governo, distribuição de casos novos por ano de diagnóstico, coeficiente de incidência e características clínicas (tipo de entrada, forma clínica da tuberculose, doenças associadas, resultado da $1^{\mathrm{a}}$ baciloscopia, resultado da $2^{\mathrm{a}}$ baciloscopia, resultado de sorologia para HIV, uso de terapia antirretroviral, realização do teste molecular rápido; realização de teste rápido de sensibilidade; tratamento diretamente observado e situação de encerramento).

A análise foi realizada através de estatística descritiva, respeitando-se assim aspectos éticos da Resolução $\mathrm{N}^{\circ}$ 510/16 (Brasil, 2016) do Conselho Nacional de Saúde, que dispõem sobre as normas aplicáveis as pesquisas, considerando que são procedimentos metodológicos que envolvem uma pesquisa com bancos de dados, cujas informações são agregadas, sem possibilidade de identificação individual, dados indiretamente obtidos com informações de acesso público, portanto não acarreta riscos à população em estudo.

Posteriormente, os dados foram submetidos à análise descritiva, buscando-se esquematizar a variabilidade dos dados entre si. Seguindo a mensuração dos dados, eles foram apresentados de forma descritivas para as frequências absoluta e relativa.

\section{Resultados}

Segundo o Levantamento Nacional de Informações Penitenciárias (Infopen) (2019), e considerando as pessoas privadas de liberdade em estabelecimentos penais e pessoas privadas de liberdade (PPL) detidos em outras carceragens, o Brasil possui uma população prisional de 773.151 pessoas em todos os regimes. Caso sejam analisados PPL custodiadas apenas em unidades prisionais, sem contar delegacias, o país detém 758.676 pessoas neste contexto.

Em Alagoas, no período de 2009 a 2018, observa-se um crescimento significativo da população privada de liberdade no sistema prisional em todos os anos, com o aumento de 267,4\% desde o início da série histórica analisada - de 4,3\% (2.379) em 2009 para 15,8 (8.788) em 2018.

Na série histórica de 2009 a 2018, observa-se a notificação/ocorrência de casos novos de tuberculose associados às pessoas privadas de liberdade (PPL) em todas as Regiões de Saúde do Estado de Alagoas. No total de casos novos de tuberculose cerca de 68,9\% das notificações ocorreram em Maceió, segundo o Sistema de Informação de Agravos de Notificação (SINAN). 
No período de 2009 a 2018, foram diagnosticados 411 casos novos de TB entre as pessoas privadas de liberdade. Em 2013, foram registrados 94 casos da doença entre PPL, o que corresponde a um coeficiente de incidência de 2072,3/100 mil hab., considerada a maior incidência no período estudado. Embora tenha sido observada uma constante tendência de queda entre os anos de 2014 e 2017, o coeficiente de incidência da TB entre PPL aumentou no ano de 2018 (Figura 1).

Figura 1: Coeficiente de incidência de tuberculose entre PPL (por 100 mil hab.), Alagoas, 2009 a 2018.

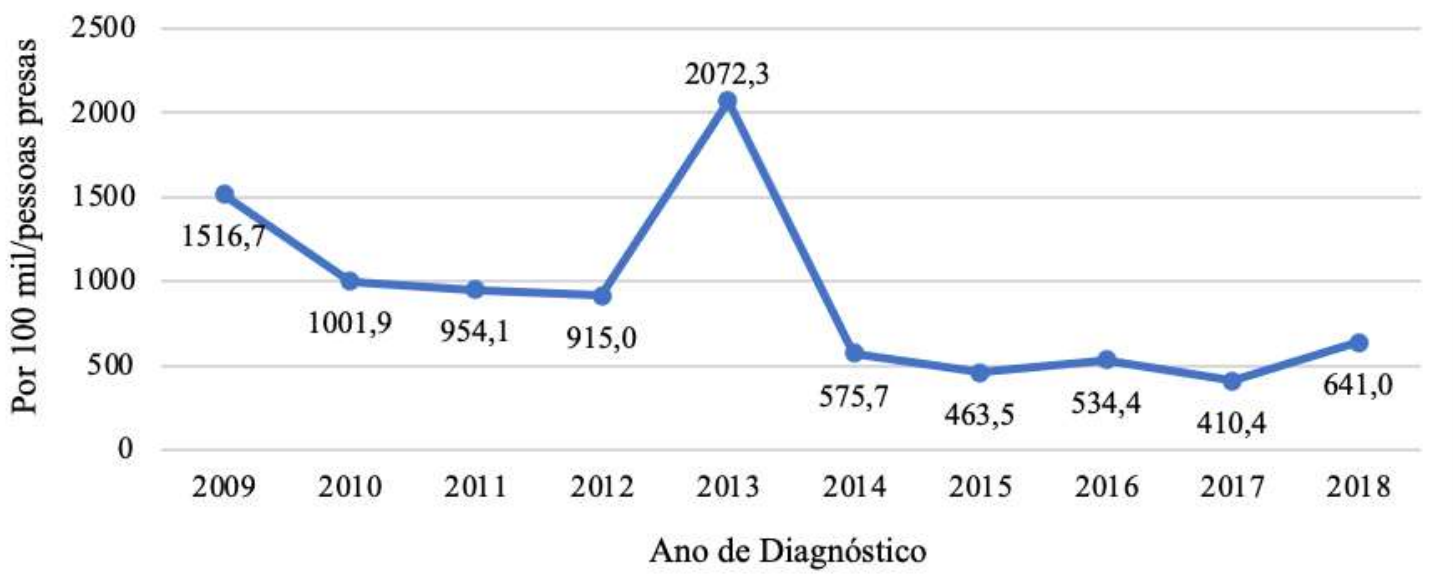

Fonte: Ministério da Saúde/SVS - Sistema de Informação de Agravos de Notificação - SINAN Net, 2020 e projeção linear da PPL de 2009 a 2018 provenientes do Infopen.

Dentre os 411 casos novos notificados de tuberculose entre as pessoas privadas de liberdade de Alagoas, constata-se que 80,8\% eram do sexo masculino. Em relação à faixa etária (em anos), o maior número de casos concentra-se entre 20 e 39 anos $(63,5 \%)$. Em relação à raça/cor da pele, 330 (80,3\%) eram da cor preta/pardos e 11,4\% eram brancos. A prevalência de pretos/pardos é cerca de oito vezes a prevalência de brancos. Na variável escolaridade, apenas 3,4\% tinham até o ensino fundamental, observando que 34,8\% dos casos estavam assinalados como "ignorado" ou não assinalados (em branco), percebendo uma possível falha no preenchimento da ficha de notificação. Ao analisar a variável beneficiário do governo, observou-se que apenas $18,7 \%$ dos casos novos possuem algum tipo de benefício (Tabela 1). 
Tabela 1: Características sociodemográficas dos casos novos $(\mathrm{n}=411)$ de tuberculose notificados entre a PPL, Alagoas, 2009 a 2018.

\begin{tabular}{|c|c|c|}
\hline Carac. Sociodemográficas & \multicolumn{2}{|c|}{ Frequência de casos } \\
\hline Sexo & $\mathbf{N}^{\mathbf{o}}$ & $\%$ \\
\hline Feminino & 79 & 19,2 \\
\hline Masculino & 332 & 80,8 \\
\hline Faixa etária (em anos) & $\mathbf{N}^{\mathbf{o}}$ & $\%$ \\
\hline 05 a 09 anos & 03 & 0,7 \\
\hline 10 a 14 anos & 07 & 1,7 \\
\hline 15 a 19 anos & 33 & 8,0 \\
\hline 20 a 39 anos & 261 & 63,5 \\
\hline 40 a 59 anos & 73 & 17,8 \\
\hline 60 a 69 anos & 21 & 5,1 \\
\hline 70 a 80 anos/sup. & 13 & 3,2 \\
\hline Raça/cor da pele & $\mathbf{N}^{\mathbf{o}}$ & $\%$ \\
\hline Ignorado/Branco & 30 & 7,3 \\
\hline Branca & 47 & 11,4 \\
\hline Preta & 54 & 13,1 \\
\hline Amarela & 04 & 1,0 \\
\hline Parda & 276 & 67,2 \\
\hline Indígena & - & - \\
\hline Escolaridade & $\mathbf{N}^{\mathbf{0}}$ & $\%$ \\
\hline Ign/Branco & 143 & 34,8 \\
\hline Analfabeto & 47 & 11,4 \\
\hline $1^{\mathrm{a}}$ a $4^{\mathrm{a}}$ série incompleta & 81 & 19,7 \\
\hline $4^{\mathrm{a}}$ série completa $\mathrm{EF}$ & 21 & 5,1 \\
\hline $5^{\mathrm{a}}$ a $8^{\mathrm{a}}$ série incompleta & 63 & 15,3 \\
\hline Ensino fundamental completo & 14 & 3,4 \\
\hline Ensino médio incompleto & 20 & 4,9 \\
\hline Ensino médio completo & 16 & 3,9 \\
\hline Ensino superior incompleto & 03 & 0,7 \\
\hline Ensino superior completo & 01 & 0,2 \\
\hline Não se aplica & 02 & 0,5 \\
\hline Beneficiário do Governo & $\mathbf{N}^{\mathbf{o}}$ & $\%$ \\
\hline Ign/Branco & 312 & 75,9 \\
\hline Não & 22 & 5,4 \\
\hline Sim & 77 & 18,7 \\
\hline
\end{tabular}

Fonte: Ministério da Saúde/SVS - Sistema de Informação de Agravos de Notificação - SINAN Net.

Em relação às características clínicas dos casos novos de tuberculose entre as pessoas privadas de liberdade (20092018), a forma clínica pulmonar esteve quase que na totalidade, representada em 90,3\% dos casos, seguida da extrapulmonar $(7,5 \%)$ e pulmonar + extrapulmonar (2,2\%). Na estratificação por ano, observa-se que na forma clínica pulmonar há uma variação no número de casos, incidindo de 6,1\% a 12,7\% (2009 e 2018), respetivamente), apresentando sua maior incidência em 2013, com 20,4\%.

Com relação a doenças e agravos associados, 70,5\% das pessoas privadas de liberdade com tuberculose, apresentaram alguma comorbidade associada. Observou-se a maior frequência de tabagismo (16,5\%), alcoolismo (14,6\%) e drogas ilícitas $(14,1 \%)$.

Ao analisar à realização da $1^{\text {a }}$ baciloscopia de escarro para BAAR (Bacilo álcool-ácido resistentes) realizada em amostra para diagnóstico, constatou-se que $65,7 \%$ das amostras tiveram resultado positivo, $14,8 \%$ negativo, $18,5 \%$ não realizado e 1,0\% das notificações com o campo não se aplica preenchido. Com relação à realização da $2^{\mathrm{a}}$ baciloscopia de escarro para BAAR (Bacilo álcool-ácido resistentes) realizada em amostra para diagnóstico, constatou-se que 32,6\% das amostras tiveram resultado positivo. 
Com relação à realização de sorologia para diagnóstico do vírus da imunodeficiência adquirida (HIV) entre as pessoas privadas de liberdade com tuberculose, identificou-se que $9,0 \%$ obtiveram resultado positivo e $24,3 \%$ não realizado

De 2009 a 2018, a proporção de casos novos de TB testados para HIV cresceu consideravelmente. No período, os achados mostram que 75,7\% dos casos novos de TB conheciam seu status para a infecção pelo HIV (Figura 2).

Figura 2: Proporção de testagem para o HIV e de coinfecção TB-HIV entre os casos novos de tuberculose, Alagoas, 2009 a 2018 .

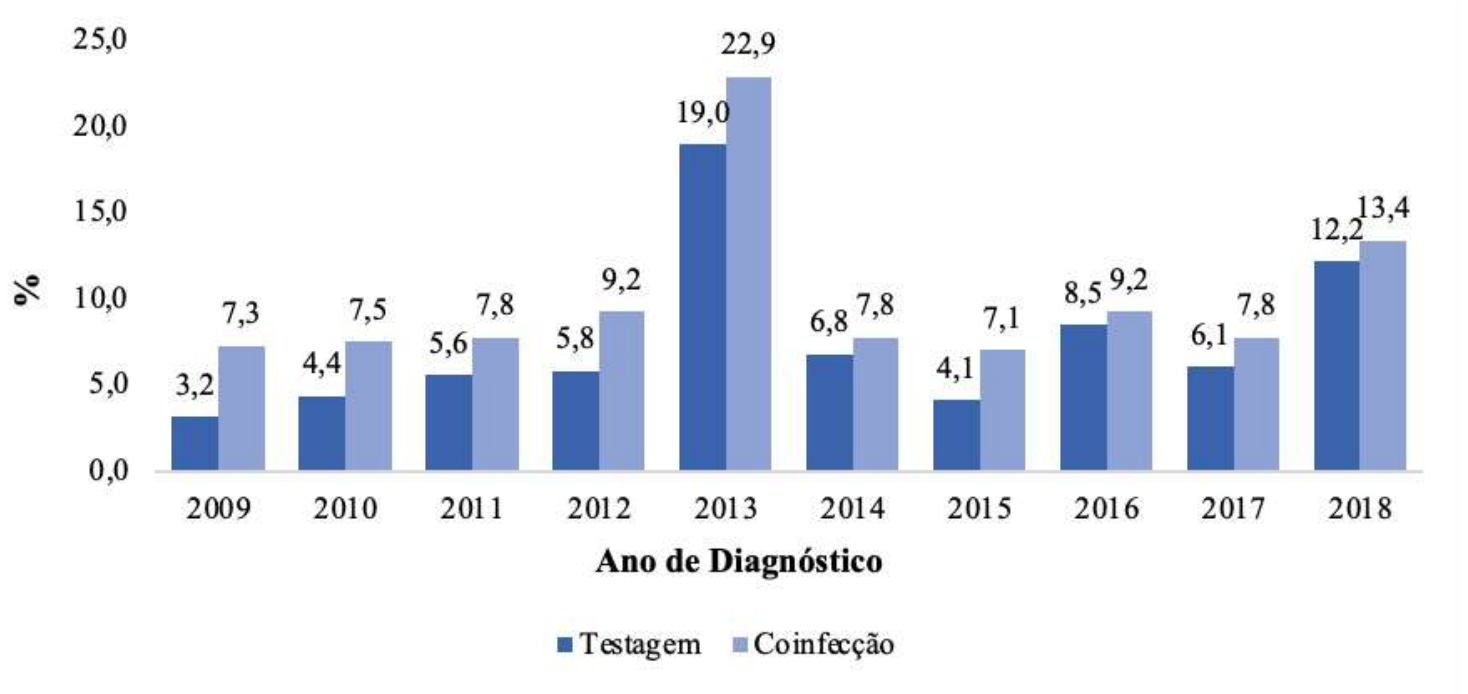

Fonte: Ministério da Saúde/SVS - Sistema de Informação de Agravos de Notificação - SINAN Net.

Especificamente, ao analisar a variável de uso de terapia antirretroviral (TARV) para HIV/Aids durante o tratamento para a tuberculose entre as pessoas privadas de liberdade, identificou-se que apenas 1,7\% das PPL com HIV/Aids fazem o uso da TARV.

Em relação à realização da cultura de escarro (qualquer material) para $M$. tuberculosis realizada em amostra para diagnóstico ou acompanhamento entre as pessoas privadas de liberdade, constatou-se que 17,5\% apresentaram resultado positivo.

Em relação à realização do teste molecular rápido (TMR-TB) realizado para M. tuberculosis em amostra para diagnóstico entre as pessoas privadas de liberdade, identificou que 20,4\% detectável sensível à rifampicina e 0,3\% detectável resistente à rifampicina.

Quanto ao resultado do teste de sensibilidade para conhecimento oportuno do padrão de resistência às drogas de $1^{\mathrm{a}}$ linha, identificou-se que em 1,0\% apresentou resistência a isoniazida, $0,2 \%$ resistência a rifampicina, $0,2 \%$ resistência a drogas de $1^{\mathrm{a}}$ linha.

Em relação ao tratamento diretamente observado (TDO) nas pessoas privadas de liberdade com tuberculose, $54,3 \%$ houve o TDO dentro da unidade prisional e $24,1 \%$ não foi realizado.

Ao analisar a situação de encerramento dos casos novos de tuberculose entre as pessoas privadas de liberdade, identificou-se que em $68 \%$ dos casos houve a cura da infecção pela tuberculose, 8,8\% abandono, 1,9\% óbito por tuberculose, $2,2 \%$ óbito por outras causas, $16,8 \%$ transferência, $1,1 \%$ tuberculose drogarresistente e 1,1\% dos casos foram assinalados o campo ignorado/branco. 


\section{Discussão}

Com base nos resultados do estudo percebeu-se algumas lacunas quanto à ausência de preenchimento de alguns campos nas fichas de notificação, como ignorados, ou até mesmo não preenchidos (em branco) o que dificultou a interpretação dos dados, entretanto não inviabilizou a análise temporal das variáveis consideradas permitindo verificar as características epidemiológicas da Tuberculose entre as PPL.

O crescente número de pessoas em situação de confinamento no sistema prisional é um dos principais fatores que agravam os problemas relacionados à saúde nesse ambiente. Segundo Reis e Bernardes (2011), as pessoas privadas de liberdade encontram-se à margem da sociedade, devido, sobretudo, às condições precárias no sistema penitenciário, além de que a atenção à saúde dessa população não equivale à proporcionalmente à população livre.

Neste sentido, superlotação das celas, estrutura física com pouca ventilação, umidade e o longo período de confinamento, são fatores observados que facilitam a proliferação do bacilo no cárcere e consequentemente leva ao processo de adoecimento entre as pessoas privadas de liberdade.

Atualmente, a saúde no sistema prisional é regida pela Política Nacional de Atenção Integral a Saúde das Pessoas Privadas de Liberdade no Sistema Prisional (PNAISP) que prevê ações voltadas à promoção da saúde da população carcerária, colaborando para o controle e prevenção de agravos' dentre eles tuberculose, hanseníase, hepatites, infecções sexualmente transmissíveis (ISTs)/Síndrome da Imunodeficiência Adquirida (Aids), além de diabetes, hipertensão e alterações psicossociais decorrentes do confinamento. A tuberculose pulmonar é discutida na maioria dos estudos sobre saúde prisional, apontando que esta doença representa um dos grandes problemas de saúde entre as pessoas privadas de liberdade(Brasil, 2004; Brasil, 2014).

Esta vulnerabilidade à tuberculose é mostrada no estudo de Santos \& Sá (2014), que revela que a PPL apresenta 27 vezes mais chances de contágio que a população livre. Para Diuana et al. (2008), a transmissão de enfermidades contagiosas constitui um sério risco não apenas aos reclusos como também a seus contatos (PPL, familiares e agentes penitenciários) e à comunidade em que serão inseridos após o seu processo de liberdade. No Brasil, há evidências de que as prisões apresentam condições favoráveis de serem amplificadoras da TB na população geral e reservatórios da doença (Sacchi et al.,2015; Mabud et al., 2019).

Mediante o registro de casos novos notificados observa-se que houve um avanço gradual, com a presença de picos nos anos de 2013, 2016 e 2018, podendo associar-se este fenômeno a intensificação de ações e estratégias de busca ativa nas unidades prisionais e uma ampliação do acesso às ferramentas de diagnóstico, com o advento da instituição do Plano Nacional de Saúde no Sistema Penitenciário em 2013, com a habilitação de equipes de atenção básica prisional com aporte de recursos financeiros federais em 2016 e a implantação do Projeto Prisões Livres de Tuberculose em 2018.

Rocha et. al., (2020), Araújo et al. (2013) e Araújo et al. (2005), afirmam que, embora de forma lenta, os números de casos da tuberculose na população geral vêm diminuindo, devendo-se isso as intensificações das notificações, maiores divulgações da doença e diagnóstico precoce. Contudo, no âmbito prisional, o contexto é diferente e com o crescimento da população carcerária, a identificação da TB em tempo oportuno é negligenciada. Todavia, é recomendado que sejam realizadas a busca ativa de sintomáticos no ingresso à unidade prisional e buscas periódicas nas celas e módulos a fim de evitar a transmissão entre as PPL (Macedo et al. 2017).

Quando analisado as características sociodemográficas, os resultados evidenciam que dentre os casos notificados das PPL com TB, a maior frequência foi do sexo masculino. Um dos fatores que explica tal realidade é que a população carcerária no Brasil é formada majoritariamente pelo público masculino, sendo o mesmo padrão no estado de Alagoas. Neste sentido, outro aspecto de relevância para atribuir-se maior susceptibilidade ao gênero é que homens que são acometidos pela infecção 
em geral possuem baixo nível de instrução, frequentemente tem dificuldade de acesso aos serviços de saúde e na prisão esta condição se agrava (Junior et al., 2013).

Com relação à faixa etária da população em questão, constata-se que os achados deste estudo corroboram com a pesquisa realizada no sistema prisional de Campinas-SP por Oliveira e Cardoso (2004), onde a maioria das PPL possuía entre 25 e 34 anos. Os valores registrados seguem o padrão nacional, demonstrando um predomínio de acometimento na faixa etária entre 20 e 49 anos, uma das fases mais ativas da vida.

Ao analisar a escolaridade, observou-se que a grande maioria das pessoas privadas de liberdade possui ensino fundamental incompleto. A baixa escolaridade ou a ausência dela na maioria dos casos é um fator de risco para a tuberculose como também para não adesão do tratamento e para aumento dos índices de abandono.

Neste sentido, a baixa escolaridade representa a reprodução de todo um contexto social que o indivíduo está inserido. Sabe-se que, a população prisional tendencialmente é advinda de condições socioeconômicas gerais precárias, provenientes de segmentos com baixas oportunidades de acesso à educação de qualidade e pouco instruídos, aumentando, assim, tanto a vulnerabilidade à tuberculose como a não adesão ao tratamento, interferindo nos desfechos negativos da doença.

No que se refere às características clínicas-epidemiológicas da tuberculose do estudo em discussão, observou-se a maior frequência de casos na forma clínica pulmonar, uma vez que autores afirmam ainda que o ambiente do confinamento age como depósito para circulação da micobactéria (Ribeiro \& Oliveira 2015).

Em relação às doenças e agravos associados, o estudo apresentou uma alta frequência de pessoas privadas de liberdade com tabagismo, etilismo e drogas ilícitas associadas. Estudos recentes apontam esses fatores de riscos para os maus resultados ao tratamento da tuberculose (Silva, et al., 2019). Associando o HIV aos casos novos de tuberculose, o estudo apresentou que não foi realizada a sorologia para diagnóstico de coinfecção HIV em todas as PPL com TB.

As pessoas privadas de liberdade no sistema prisional têm um risco aumentado para a coinfecção tuberculose/HIV que implica um risco anual de desenvolver a doença, semelhante ao risco vitalício estimado para indivíduos infectados apenas pelo bacilo de Koch.

Segundo Sacchi et al., (2015), a Aids é uma das comorbidade que apresentam maiores fatores de risco para a TB. É sabido que o HIV ocasionou alterações nos mecanismos de defesa do organismo humano contra o agente causal da TB, por conta disso a infecção pelo HIV pode ser dita como o principal fator de risco para a evolução da infecção em latência provocada pelo M. tuberculosi.

Um aspecto importante apresentado no estudo é o uso de terapia antirretroviral (TARV) para HIV/Aids durante o tratamento para a tuberculose entre as pessoas privadas de liberdade, onde nem todas as PPL estavam em tratamento. O tratamento da tuberculose deve ser priorizado e o tratamento com antirretroviral deve ser instituído em até 8 semanas após o início do tratamento da TB (Brasil, 2019).

O tratamento para tuberculose deverá ser instituído imediatamente e os antirretrovirais devem ser introduzidos de acordo com o grau de imunossupressão avaliado através da contagem de LT-CD4+ (Brasil, 2017).

Em relação ao tratamento diretamente observado, os dados encontrados revelam uma alta frequência de PPL que não fazem TDO. Segundo Diuana et. al. (2008), o tratamento de tuberculose nas PPL deve ser diretamente observado e realizado exclusivamente por profissionais de saúde, para que seja possível possibilitar momentos de troca de informações, estabelecimento do cuidado, diferenciando-se de medidas de controle e fiscalização que prevalecem nas prisões.

Neste estudo observou-se uma alta frequência de abandono ao tratamento entre as pessoas privadas de liberdade, considerado superior ao estimado pelo MS, uma vez que a taxa de abandono recomendada pela OMS é de até 5\%.

A taxa de abandono é um fator importante, pois o abandono do tratamento tem sido repetidamente um considerável fator que favorece a manifestação de bacilos multirresistentes como também maior barreira para controle e eliminação da 
doença (Paz et al., 2012). Outro considerável fator prevalente que interfere diretamente na proporção de cura, aumentando os casos de encerramento por transferência, além das condições precárias de infraestrutura das prisões, é a alta rotatividade das pessoas privadas de liberdade (Telisinghe et al., 2014).

Ademais, o sistema prisional é repleto de especificidades que implicam na atenção integral à saúde das PPL, necessitando da compreensão das características epidemiológicas desta população para traçar estratégias de cuidado com vistas na redução das fontes de infecção e no controle da transmissão.

\section{Conclusão}

Os resultados deste estudo contemplaram o objetivo proposto e levaram à reflexão sobre a necessidade de melhoria na qualidade do cuidado prestado a este público, considerando o crescimento acelerado da população privada de liberdade, pois se não lançarmos estratégias específicas no contexto prisional e tratarmos os fatores de risco para adoecimento da tuberculose, será difícil pensar no fim da tuberculose ou até mesmo no seu controle.

Para isso, é necessário o estabelecimento de novas medidas e a ampliação de estratégias de cuidado, de acordo com a realidade epidemiológica local, visando romper barreiras de detectar, notificar, minimizar e prevenir situações de exposição e aumento de casos novos entre as pessoas privadas de liberdade, além de direcionar em tempo oportuno o cuidado integral a PPL infectada, bem como reduzir os riscos de transmissão da infecção no cárcere. Além disso, os dados contemplados nesta pesquisa permitem suporte para futuras pesquisas práticas de promoção e prevenção deste agravo no contexto da pessoa privada de liberdade com subsídio científico para as futuras ações voltadas para este público.

\section{Referências}

Araújo, K. M. F. A., Figueiredo, T. M. R. M., Gomes, L. C. F, Pinto, M. L., Silva, T. C. \& Bertolozzi, M. R. (2013) Evolution of the spatial distribution of tuberculosis cases in the city of Patos (PB), 2001-2010. Cad Saúde Colet., 21(3):296-302.

Araújo, S. R. L., Pereira I. S. S.D., Brito N. O. S. \& Fonseca P. C. B. (2005). Perfil epidemiológico da tuberculose pulmonar na cidade de Natal - RN. J Infect Control, 4(1):16-19.

Brasil (2004). Ministério da Saúde. Secretária de Atenção à Saúde. Departamento de Ações Programáticas Estratégicas. Área Técnica de Saúde no Sistema Penitenciário. Plano Nacional de Saúde no Sistema Penitenciário. Brasília: Ministério da Saúde.

Brasil (2014). Ministério da Saúde. Portaria Interministerial no 1/GM, de 2 de janeiro de 2014. Institui a Política Nacional de Atenção Integral à Saúde das Pessoas Privadas de Liberdade no Sistema Prisional (Pnaisp) no âmbito do Sistema Único de Saúde (SUS).

Brasil (2014). Ministério da Saúde. Secretaria de Vigilância em Saúde. Boletim Epidemiológico da tuberculose. 44, 2014 b.

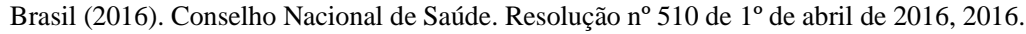

BRASIL (2017). Ministério da Saúde. Boletim Epidemiológico: Indicadores prioritários para o monitoramento do Plano Nacional pelo Fim da Tuberculose como Problema de Saúde Pública no Brasil. v. 48, n. 8. Secretaria de Vigilância em Saúde - Ministério da Saúde - Brasil.

Brasil (2019). Ministério da Saúde. Secretaria de Vigilância em Saúde. Departamento de Vigilância das Doenças Transmissíveis. Manual de Recomendações para o Controle da Tuberculose no Brasil / Ministério da Saúde, Secretaria de Vigilância em Saúde, Departamento de Vigilância das Doenças Transmissíveis. - Brasília: Ministério da Saúde.

Diuana V., Lhuilier, D., Sánchez, A. R., Amado, G., Araújo, L, Duarte, A. M., Garcia, M., Milanez, E., Poubel, L., Romano, E. \& Larouzé, B (2008). Saúde em prisões: representações e práticas dos agentes de segurança penitenciária no Rio de Janeiro, Brasil. Cad Saúde Pública,24(8):1887-1896.

Hargreaves, J. R., Boccia, D., Evans, C. A., Adato, M., Petticrew, M. \& Porter, J. D. (2011) The Social Determinants of Tuberculosis: From Evidence to Action. American Journal of Public Health, 101(4):654-662.

Junior, S. F., Oliveira, H. B. \& León, L. M. (2013). Conhecimento, atitudes e práticas sobre tuberculose em prisões e no serviço público de saúde. Rev Bras Epidemiol., 16(1):100-113.

Mabud, T. S., Alves, M. L. D., Ko, A. I., Basu, S., Walter, K. S., Cohen, T., Mathema, B., Colijn, C., Lemos, E., Croda, J. \& Andrews, J. R. (2019). Evaluating strategies for control of tuberculosis in prisons and prevention of spillover into communities: Na observational and modeling study from Brazil. PLOS Med., 16(1).

Macedo, L. R., Maciel, E. L. N. \& Struchiner, C. J. (2017). Tuberculose na população privada de liberdade no Brasil, 2007-2013. Epidem Serv Saúde., 26(4):783-974. 
Research, Society and Development, v. 11, n. 1, e22411124903, 2022

(CC BY 4.0) | ISSN 2525-3409 | DOI: http://dx.doi.org/10.33448/rsd-v11i1.24903

Navarro, P. D., Almeida, I. N., Kritski, A. L., Ceccato, M. G., Maciel, M. M. D., Carvalho, W. S. \& Miranda, S. S. (2016) Prevalence of latent Mycobacterium tuberculosis infection in prisoners. J Bras de Pneumol, 42(5): 348-355.

Oliveira, H. B. \& Cardoso, J. C. (2004). Tuberculosis among city jail inmates in Campinas, São Paulo, Brazil. Rev Panam Salud Publica, 15(3):185-193.

Paz, L. N. F., Ohnishi, M. D. O., Barbagelata, C. M., Bastos, F. A., Oliveira, J. A. F. \& Parente, I. C. (2012). Efetividade do tratamento da tuberculose. J Bras Pneumol, 38(4): 503-510.

Reis C. B. \& Bernardes E. B. (2011) O que acontece atrás das grades: estratégias de prevenção desenvolvidas nas delegacias civis contra HIV/AIDS e outras doenças sexualmente transmissíveis. Cienc Saude Coletiva, 16(7):3331-3338.

Ribeiro, R. C. \& Oliveira, C. G. (2015). As mazelas do sistema prisional brasileiro. Rev. Jurídica, 24(1):113-128.

Rocha, M. S., Bartholomay, P., Cavalcante, M. V., Medeiros, M. C., Codenotti, S. B. \& Pelissari, D. M., et al. (2020). Sistema de Informação de Agravos de Notificação (Sinan): principais características da notificação e da análise de dados relacionada à tuberculose. Epidemiologia e Serviços de Saúde. 29 (1): 1-13.

Sacchi, F. P. C., Praça, R. M., Tatara, M. B., Simonsen, V., Ferrazoli, L, Croda, M. G., Suffys, P. N., Ko, A. I., Andrews J. R. \& Croda, J. (2015). Prisons as reservoir for community transmission of tuberculosis, Brazil. Emerg Infect Dis., 21(3):452-455.

Santos, M. N. A. \& Sá, A. M. M. (2014). Viver com tuberculose em prisões: o desafio de curar-se. Texto contexto - enferm., 23(4):854-61.

Silva, B. N., Temoteo, R. C. A., Véras, G. C. B. \& Silva, C. R. D. V. (2019) Fatores predisponentes de tuberculose em indivíduos privados de liberdade: revisão integrativa. Arch. Health. Sci., 26(1): 67-71.

Silva, S. A., Silva, G. C., Nascimento, G. C. R., Rocha, M. V. C. \& Oliveira, K. C. P. N. (2021). Prison system and life conditions of the man in prison in a region of brazilian northeast. Research, Society and Development, 10(7):1-10.

Telisinghe, L., Fielding, K. L., Malden, J. L., Hanifa, Y., Churchyard, G. J., Grant, A. D. \& Charalambous, S. (2014). High Tuberculosis Prevalence in a South African Prison: The Need for Routine Tuberculosis Screening. PLoS ONE, 9(1).

WHO. (2014) World Health Organization. Global tuberculosis report 2014. Geneva: World Health Organization. 